\title{
Metabolomics tools for the synthetic biology of natural products
}

DOI:

10.1016/j.copbio.2018.02.015

\section{Document Version}

Accepted author manuscript

Link to publication record in Manchester Research Explorer

\section{Citation for published version (APA):}

Hollywood, K., Schmidt, K., Takano, E., \& Breitling, R. (2018). Metabolomics tools for the synthetic biology of natural products. Current Opinion in Biotechnology, 54, 114-120. https://doi.org/10.1016/j.copbio.2018.02.015

\section{Published in:}

Current Opinion in Biotechnology

\section{Citing this paper}

Please note that where the full-text provided on Manchester Research Explorer is the Author Accepted Manuscript or Proof version this may differ from the final Published version. If citing, it is advised that you check and use the publisher's definitive version.

\section{General rights}

Copyright and moral rights for the publications made accessible in the Research Explorer are retained by the authors and/or other copyright owners and it is a condition of accessing publications that users recognise and abide by the legal requirements associated with these rights.

\section{Takedown policy}

If you believe that this document breaches copyright please refer to the University of Manchester's Takedown Procedures [http://man.ac.uk/04Y6Bo] or contact uml.scholarlycommunications@manchester.ac.uk providing relevant details, so we can investigate your claim.

\section{OPEN ACCESS}




\title{
Metabolomics tools for the synthetic biology of natural products
}

\author{
Katherine A. Hollywood, Kamila Schmidt, Eriko Takano and Rainer Breitling
}

Manchester Centre for Fine and Speciality Chemicals (SYNBIOCHEM), Manchester Institute of Biotechnology, School of Chemistry, Faculty of Science and Engineering, The University of Manchester, Manchester M1 7DN.

\begin{abstract}
Metabolomics plays an increasingly central role in within the Design - Build - Test cycle of synthetic biology, in particular in applications targeting the discovery, diversification and optimized production of a wide range of natural products. For example, improved methods for the online monitoring of chemical reactions accelerate data generation to be compatible with the rapid iterations and increasing library sizes of automated synthetic biology pipelines. Combinations of label-free metabolic profiling and ${ }^{13} \mathrm{C}$-based flux analysis lead to increased resolution in the identification of metabolic bottlenecks affecting product yield in engineered microbes. And molecular networking strategies drastically increase our ability to identify and characterize novel chemically complex biomolecules of interest in a diverse range of samples.
\end{abstract}

\section{Graphical abstract}

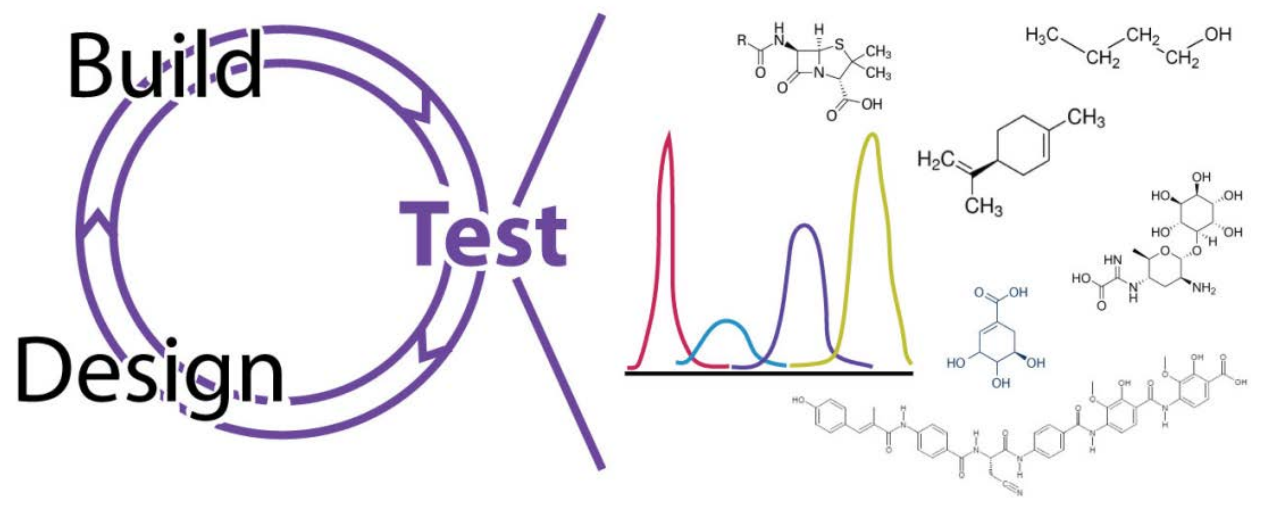

\section{Synthetic biology of natural products}

Synthetic biology facilitates the biosynthesis of pharmaceutical ingredients and other high-value chemicals by employing the Design - Build - Test cycle of engineering to guide the systematic enhancement of microbial factories [1-4]. Exemplary successful applications of synthetic biology to natural product production include a one-pot method for menthol biosynthesis in Escherichia coli [5], the modular extension of a styrene biosynthesis pathway to produce 2phenylethanol [6], cannabinoid biosynthesis in yeasts [7], and the heterologous production of antibiotics using extensively refactored biosynthetic gene clusters: myxobacterial $\alpha$-pyrone antibiotics in Myxococcus xanthus [8] and kasugamycin (an aminoglycoside antibiotic isolated from Streptomyces kasugaensis) in actinomycetes [9]).

A recent review by Smanski and colleagues [10] provides details of recent advances in the technologies underpinning the Build aspects of the synthetic biology cycle, including pathway construction and pathway screening, while a complementary review by Chen et al. [11] focuses 
on the modelling approaches for the construction and optimisation of cell factories for bioproduction, which cover a large part of the Design activities. In the present review, we will in turn focus on the Test component of synthetic biology, focusing in particular on advances in metabolomics as a discovery and debugging tool for metabolically enhanced microbial systems.

\section{Test Analytics - Appropriate Technologies}

Mass spectrometry (MS) coupled to chromatography remains the domineering technology used for the quantification of natural product targets and is also the most widely used platform for the global profiling of the impact of an engineered biosynthetic pathway on the microbial metabolome. The challenge for the analytical technologies is to achieve the acquisition speed and sensitivity required to meet the high-throughput needs of a synthetic biology-based pipeline. Traditionally, products are measured directly from an aliquot of cell culture medium or - in the case of volatile products - they are captured in solvent overlays and transferred to vials or multi-well plates for analysis. These approaches are often slow (tens of minutes per sample plus preparation time) and provide only a snap shot of what is occurring at a given time.

To overcome this limitation, much effort has been invested into the development of improved methods for the online monitoring of chemical reactions; which would provide greater control of sampling and provide dynamic results with regards to product turnover. Definitions of the analytical terminology described herein are summarised in Table 1. In recent work by Yan et al., desorption electrospray ionization (DESI) coupled to ion-mobility mass spectrometry was used for the high-throughput screening of biocatalysis directly from bacterial colonies on agar plates [12], which can in principle be applied to a broad range of substrates and products, including free amines, carboxylic acids, alkaloids and phenols; multiple analytes can be detected in a single analysis thus allowing for the screening of diverse strain libraries with complex product profiles. DESI-MS was also applied to the rapid analysis of enzyme kinetics by Cheng and coworkers [13], who measured product formation in a buffered aqueous medium, explored the possibility of adjusting the $\mathrm{pH}$ and solvent composition of the DESI spray to quench the enzymatic reaction and thus improved the accuracy of the kinetic measurements by preventing post-ionization reactions.

As an alternative to DESI, matrix-assisted laser desorption ionisation mass spectrometry imaging (MALDI-MSI) has readily been applied towards the large-scale phenotyping of bacteria $[14,15]$. A related optically-guided MALDI-MS strategy has recently been implemented for the profiling of microbial colonies for rapid screening of natural product analogue libraries [16]. This impressive development used optical imaging of microbial colonies to direct the laser coordinates for an automated MALDI-MS screening of approximately 1000 colonies directly from an imprinted glass slide with an MS sampling rate of about one colony per second. Reaction products were screened in situ and results overlaid with the optical images; integration of results allowed for subsequent colony picking and recovery of the desired mutant strains. The majority of commercially available MALDI-MS instrumentation permit a spatial resolution of $>100 \mu \mathrm{m}$. However, the group of Bernhard Spengler has recently dramatically pushed this boundary towards much better lateral resolutions down to $1.4 \mu \mathrm{m}$ [17], thus further advancing the technique towards single cell resolution and even higher throughput [18].

The coupling of microreactors or continuous flow chemical reactors directly to the mass spectrometer provides an enhanced ability to characterise unstable reaction products and reduces the sample volume required (albeit with sufficient mass spectrometer sensitivity). Link et al. [19] provided a comprehensive example of such an application. They demonstrated the ability to undertake real-time metabolome profiling by direct 
injection of living bacteria, yeast and mammalian cells into a high-resolution mass spectrometer through coupling a peristaltic pump and two six-port valves and automatically sampling from a liquid culture. This approach permitted the automated monitoring of around 300 compounds in 15-30 s cycles over several hours. They investigated the metabolite dynamics in real-time during $2 \mathrm{~h}$ starvation and $30 \mathrm{~min}$ of growth resumption. The approach suggested that the accumulation of energetically costly metabolites in starved $E$. coli reflects the control strategy to favour cheap metabolic pathways for growth resumption. From an analytical perspective the method permitted real-time metabolome profiling that followed the dynamics of metabolic processes in different organisms over extended periods. The method alleviates retrospective manual sampling, sample preparation and sample manipulation associated with traditional off-line methods.

Progress has also been made on the mass spectrometry techniques available to the synthetic biology community: proton transfer reaction mass spectrometry (PTR-MS) and selected ion flow tube mass spectrometry (SIFT-MS). These techniques are direct injection approaches that utilise chemical ionisation for real time analysis of volatile organic compounds. PTR-MS has been shown to achieve near-to-real-time monoterpene separation and identification, when coupled to a fast gas chromatography, with sensitivity in the range of $1.2 \mathrm{ppbv}$ from plant material [20]. PTR-MS has also been applied to the real time monitoring of the yeast volatilome [21], detecting more than 300 metabolite features, 70 of which were tentatively identified, in the headspace of Saccharomyces. cerevisae cultures over 11 days at 4-h time points. Additional development and application of this technique has been demonstrated by Materic et al. [22], who used Selective Reagent Ion PTR-MS to investigate the separation of monoterpene mixtures, which are a particularly common target in recent synthetic biology projects i.e. geraniol [23], linalool [24] and limonene [25].

\section{Global analysis - Metabolomics}

Synthetic biology requires not only the rapid and accurate quantitation of the desired end products; even more important for a systematic engineering of the microbial factories is a thorough understanding of metabolic flux and the regulation of central carbon metabolism to ensure the desired production of target compounds is compatible with maintaining cellular homeostasis and energy balance. Metabolomics, the comprehensive profiling of small molecules in a biological sample, is the obvious method of choice for collecting the necessary data for this kind of analysis, and synthetic biology can build on a continuously refined repertoire of metabolomics approaches $[26,27]$.

Of the many technological advances in recent years, we only highlight the increasing importance of parallel reaction monitoring (PRM) in metabolomics; the quantitation of intermediates of central carbon metabolism, amino acids and shikimate pathway-related metabolites in engineered strains of E. coli [28] is just one important example of its application in synthetic biology. PRM permits the quantitative analysis of multiple targets (237 in this example) [29] with excellent linearity of quantitation, as well as high precision and accuracy. In a related approach, all ion fragmentation acquisition has recently been demonstrated to achieve increased accuracy in metabolite identification for a large number of pre-selected compounds, while at the same time acquiring full scan information to allow the identification of additional metabolites that were initially not targeted [30].

A metabolomics-driven approach was applied to identify non-obvious target genes to further improve the production of 1-butanol [31,32]. The authors performed quantitative targeted analysis of acyl-CoAs in the CoA-dependent 1-butanol biosynthetic pathway in Synechococcus elongatus strains via ${ }^{13} \mathrm{C}$-labelling of cell extracts as an internal standard and HPLC-MS analysis. The results indicated several targets for potential improvements of 1-butanol production in 
cyanobacteria, such as possible rate-limiting steps (reductive reaction of butanoyl-CoA to butanal) or effective regeneration of free-CoA from butanoyl-CoA to enhance the conversion of pyruvate to acetyl-CoA. In a parallel study addressing 1-butanol production in E. coli, the authors examined the metabolomic impact of the deletion of phosphate acetyltransferase, which was performed in an attempt to reduce the amount of acetate produced and simultaneously increase the acetyl-CoA pool. Metabolomics analysis using a targeted ion pair LC-MS/MS method detected a total of 78 metabolites and pointed to several metabolic perturbations caused by the deletion that seemed to be the consequence of a CoA imbalance or insufficient CoA recycling, which caused the undesirable accumulation of side products. Further metabolomics analysis identified the underlying enzymatic bottleneck, alcohol dehydrogenase, and fine-tuning of this activity resolved the CoA imbalance and led to substantially improved 1butanol titres [31].

A metabolomics approach was also implemented to investigate central metabolism of a fructose repressor $($ fru $R$ ) knockout in a recombinant L-tryptophan producing strain of E. coli (E. coli FB04) [33]. The authors report more than 80 intracellular metabolites that were altered as a result of the knockout, 23 of which were related to tryptophan biosynthesis. The levels of glycolysis, pentose phosphate and TCA cycle intermediates were consistently increased, and levels of shikimate derivatives (direct tryptophan precursors) and L-glutamine were decreased in the knockout strain, which also showed a substantially increased tryptophan production. The interpretation of these results illustrates very clearly the pitfalls of using steady-state metabolome profile information as a proxy for metabolic fluxes, which are of central interest for synthetic biology: based on increased levels of glycolytic and pentose phosphate pathway intermediates, the authors conclude that the fruR knockout enhanced metabolic flow through these two pathways which provide the substrates for L-tryptophan biosynthesis. However, the TCA cycle, which directly competes with tryptophan biosynthesis shows an equally increased level of its intermediates, and the only pathway for which direct flux measurements are available, tryptophan biosynthesis itself, shows a consistent decrease in its key intermediates, despite an increase in flux by $62.5 \%$ (from 0.024 to $0.039 \mathrm{~g} / \mathrm{L} / \mathrm{h}$ ).

A subsequent study combining metabolomics and ${ }^{13} \mathrm{C}$ fluxomics provided more detailed insights into the metabolic flux redistribution in an E. coli strain overproducing shikimic acid with high titres and yields: Rodriguez et al. [34] used an engineered AR36 E. coli strain constitutively expressing six proteins encoded in a synthetic operon promoting high-yield production of shikimic acid from glucose. Comparative metabolomics of a production strain and parental strains (carrying either no plasmid or "empty plasmid") was used to track the levels of seven exometabolites and 25 endometabolites over time. It revealed a global remodelling of carbon and energy metabolism in the high producer. This resulted in reduced carbon available for oxidative and fermentative pathways and increased levels of endometabolites involved in energy pathways, preventing the depletion of essential intermediates, such as PEP and ATP. Both glycolytic flux and TCA cycle activity were substantially reduced in this overproduction scenario ( $43 \mathrm{~g} / \mathrm{L}$ of shikimate in $30 \mathrm{~h}$ on complex medium).

Given its importance as a provider of essential precursors for a diverse range of biotechnologically important biochemicals, it is not surprising that the shikimate pathway has been the target of dedicated metabolomics method development: e.g., Lai et al. [35] contributed a robust HPLC method for the quantification of aromatic substrates, products and pathway intermediates in order to accelerate strain engineering for industrial production of aromatics as biosynthetic molecules. The achieved limits of detection between $10^{-10}-10^{-13} \mathrm{~mol}$ make the method suitable for endometabolome and exometabolome analysis of engineered strains.

Another example of a metabolomics-based strategy for strain engineering (this time utilising a GC-MS analytic platform) is the study by Teoh et al. [36] investigating phenotypic differences in 
growth rates and metabolite profiles of nineteen single-deletion $S$. cerevisiae mutant strains cultivated under stress-free and under 1-butanol stress conditions (growth inhibition caused by higher alcohols (e.g. 1-butanol) is considered as a bottleneck in their biosynthetic production). Metabolites associated with improved growth rates under stress conditions were identified, and new stress-resistant mutant yeast strains were successfully predicted based on their metabolite profiles. This approach illustrates the potential of metabolomics as a predictive screening tool to inform semi-rational strain engineering approaches.

Finally, metabolomics has been applied for the monitoring of isoprenoid precursors production, another classic target for synthetic biology [37, 38]. In a study by Kirby et al. [39], who report for the first time the functional expression of an extensively engineered functional 1-deoxy-Dxylulose 5-phosphate (DXP) pathway in S. cerevisiae which normally utilizes the mevalonate pathway, which has a lower theoretical yield. Metabolite-guided DXP pathway balancing, by LCMS quantification of intermediates in cultures exhibiting various levels of flux, appeared to be a successful approach for identifying a bottleneck in the pathway. An engineered strain exclusively using the DXP pathway achieved an endpoint biomass $80 \%$ of that of the same strain using the mevalonate pathway under low aeration conditions.

\section{Molecular networking - moving forward}

The main challenge of untargeted metabolomics is compound annotation; the persistent difficulties of confidently identifying the detected metabolites currently seriously limits the utility of the MS data acquired. Molecular networking, a visualisation method for tandem MS data, is a powerful complement to traditional de-replication methods [40]. This approach allows for the detection of sets of spectra from related molecules ("spectral networks"), even in the cases when these spectra are not matched to any known compounds. The approach is based on the assumption that similar molecules have similar MS fragmentation patterns so they will tend to cluster closely within a network. Each spectrum (ideally derived from a single compound) is visualised as a network node, and the edges between nodes represent a degree of similarity between spectra. The thicker the line, the more MS/MS fragment ions are shared by the two connected nodes. Nodes can be supplemented by such information as a compounds abundance, biochemical activity, origin etc. Molecular networking led to the development of Global Natural Products Social Molecular Networking (GNPS), a metabolomic data-driven platform for the storage, sharing, analysis, and knowledge dissemination of tandem MS spectra where one is able to annotate natural product data via continuous de-replication. [41].

Although improvements are still required to obtain unambiguous analysis of molecular networks such as efficient integration with existing LC-MS detection strategies, enhancement of pre-processing and universal optimal acquisition methods [40, 42, 43], its applications are expanding fast [44-49] and it will soon become an indispensable metabolomics tool in exploratory analyses for the synthetic biology of novel natural products. For example, in a recent study Crüseman and colleagues [46] screened 146 marine Salinispora and Streptomyces strains using HPLC-MS/MS, molecular networking, and the Global Natural Products Social (GNPS)[41] platform and explored the impact of differing culturing and extraction techniques. The systematic investigation of the effect of these parameters clearly demonstrated how much inherent chemical diversity could be missed when just one culture and extraction protocol is utilised to assess metabolic capacity. This example demonstrated how the application of molecular networking permits the rapid optimisation of experimental parameters that can subsequently be implemented early inthe discovery workflow.

Okada et al. [50] used molecular networking for the investigation of the influence of trimethoprim (Tmp) antibiotic on the secreted metabolome of Burkholderia thailandensis E264. The untargeted comparison of Tmp-induced and uninduced samples (utilising HPLC-QToF- 
MS/MS) resulted in $\sim 240$ metabolites of interest (with $>100$ compounds observed only for the induced samples). Organising them into 14 sub-networks followed by NMR analysis enabled rapid identification of 40 compounds including analogues of known compounds and a group of new molecules, acybolins, showing that molecular networking aids rapid identification of compounds compared to traditional workflows.

In related work, von Eckardstein et al. [47] used bioactivity-guided untargeted LC-MS/MS analysis and molecular networking in the search of new antibiotic agents from Xanthomonas albilineans. Over 20,000 MS/MS spectra acquired from crude extracts and bioactive fractions were organised into a molecular network via the GNPS portal, which allowed for the identification of potential derivatives in the albicidin sub-network. The group reported eight new natural albicidin derivatives with unambiguous identification.

\section{Conclusions}

There are still some challenges to overcome, but both synthetic biology and metabolomics are very dynamic fields that are forging an ever-closer alliance. An example that illustrates the integral role of metabolomics in synthetic biology pipelines is the recently published multiomics workflow to characterise strain variation in engineered E. coli [51]. It is certain that in coming years we will see a rapid deepening of the technical and conceptual integration of metabolic profiling methods within the Design - Build - Test cycle, and in particular the emergence of additional tools to facilitate the flow of data and insights between the analytical machinery (Test) and its users in the Design and Build stages of strain engineering.

\section{Acknowledgements}

279 Funding: KAH, ET and RB acknowledge the funding from the Biotechnology and Biological 280 Sciences Research Council (BBSRC) under grant BB/M017702/1, "Centre for synthetic biology of fine and speciality chemicals (SYNBIOCHEM)". This is a contribution from the Manchester Centre for Synthetic Biology of Fine and Speciality Chemicals (SYNBIOCHEM). KS, ET and RB received funding from the European Union's Horizon 2020 Research and Innovation Programme under Grant Agreement No. 720793 "Thoroughly Optimised Production Chassis for Advanced Pharmaceutical Ingredients (TOPCAPI).

\section{References and recommended reading}

Papers of particular interest, published within the period of review, have been highlighted as:

- of special interest

•• of outstanding interest

1. Breitling, R. and E. Takano, Synthetic biology advances for pharmaceutical production. Curr Opin Biotechnol, 2015. 35: p. 46-51.

2. Breitling, R. and E. Takano, Synthetic Biology of Natural Products. Cold Spring Harb Perspect Biol, 2016. 8(10).

3. Ren, H., P. Hu, and H. Zhao, A plug-and-play pathway refactoring workflow for natural product research in Escherichia coli and Saccharomyces cerevisiae. Biotechnol Bioeng, 2017. 114(8): p. 1847-1854.

4. Smanski, M.J., et al., Meeting Report for Synthetic Biology for Natural Products 2017: The Interface of (Meta)Genomics, Machine Learning, and Natural Product Discovery. ACS Synth Biol, 2017. 6(5): p. 737-743.

5. Toogood, H.S., et al., Enzymatic Menthol Production: One-Pot Approach Using Engineered Escherichia coli. Acs Synthetic Biology, 2015. 4(10): p. 1112-1123. 
6. Machas, M.S., R. McKenna, and D.R. Nielsen, Expanding Upon Styrene Biosynthesis to Engineer a Novel Route to 2-Phenylethanol. Biotechnology Journal, 2017. 12(10).

7. Zirpel, B., et al., Engineering yeasts as platform organisms for cannabinoid biosynthesis. Journal of Biotechnology, 2017. 259: p. 204-212.

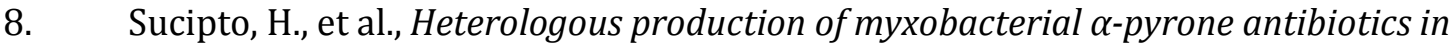
Myxococcus xanthus. Metabolic Engineering, 2017. 44: p. 160-170.

9. Kasuga, K., et al., Heterologous production of kasugamycin, an aminoglycoside antibiotic from Streptomyces kasugaensis, in Streptomyces lividans and Rhodococcus erythropolis L88 by constitutive expression of the biosynthetic gene cluster. Applied Microbiology and Biotechnology, 2017. 101(10): p. 4259-4268.

10. Smanski, M.J., et al., Synthetic biology to access and expand nature's chemical diversity. Nature Reviews Microbiology, 2016. 14(3): p. 135-149.

11. Chen, P.W., M.K. Theisen, and J.C. Liao, Metabolic systems modeling for cell factories improvement. Current Opinion in Biotechnology, 2017. 46: p. 114-119.

12. Yan, C.Y., et al., Real-Time Screening of Biocatalysts in Live Bacterial Colonies. Journal of the American Chemical Society, 2017. 139(4): p. 1408-1411.

13. Cheng, S., et al., Online Monitoring of Enzymatic Reactions Using Time-Resolved Desorption Electrospray Ionization Mass Spectrometry. Analytical Chemistry, 2017. 89(4): p. 2338-2344.

14. Zhang, L., S. Smart, and T.R. Sandrin, Biomarker-and similarity coefficient-based approaches to bacterial mixture characterization using matrix-assisted laser desorption ionization time-of-flight mass spectrometry (MALDI-TOF MS). Scientific Reports, 2015. 5.

15. AlMasoud, N., et al., Classification of Bacillus and Brevibacillus species using rapid analysis of lipids by mass spectrometry. Analytical and Bioanalytical Chemistry, 2016. 408(27): p. 7865-7878.

$\bullet \bullet 16$. Si, T., et al., Profiling of Microbial Colonies for High-Throughput Engineering of Multistep Enzymatic Reactions via Optically Guided Matrix-Assisted Laser Desorption/Ionization Mass Spectrometry. Journal of the American Chemical Society, 2017. 139(36): p. 1246612473.

In this article, the authors show how a optically-guided MALDI-MS allows the rapid identification and recovery of desirable strains from large populations of microbial colonies.

17. Kompauer, M., S. Heiles, and B. Spengler, Atmospheric pressure MALDI mass spectrometry imaging of tissues and cells at 1.4-mu m lateral resolution. Nature Methods, 2017. 14(1): p. 90-96.

18. Comi, T.J., et al., Categorizing Cells on the Basis of their Chemical Profiles: Progress in Single-Cell Mass Spectrometry. Journal of the American Chemical Society, 2017. 139(11): p. 3920-3929.

19. Link, H., et al., Real-time metabolome profiling of the metabolic switch between starvation and growth. Nature Methods, 2015. 12(11): p. 1091-1097.

20. Materic, D., et al., Monoterpene separation by coupling proton transfer reaction time-offlight mass spectrometry with fastGC. Analytical and Bioanalytical Chemistry, 2015. 407(25): p. 7757-7763.

-21. Khomenko, I., et al., Non-invasive real time monitoring of yeast volatilome by PTR-ToF-MS. Metabolomics, 2017. 13(10).

This study illustrates the high-throughput on-line monitoring of the volatile metabolome of microbial colonies enabled by automatic proton transfer reaction mass spectrometry.

22. Materic, D., et al., Selective reagent ion-time of flight-mass spectrometry study of six common monoterpenes. International Journal of Mass Spectrometry, 2017. 421: p. 40-50.

23. Jiang, G.Z., et al., Manipulation of GES and ERG20 for geraniol overproduction in Saccharomyces cerevisiae. Metabolic Engineering, 2017. 41: p. 57-66. 
24. Amiri, P., et al., Metabolic engineering of Saccharomyces cerevisiae for linalool production. Biotechnology Letters, 2016. 38(3): p. 503-508.

25. Jongedijk, E., et al., Capturing of themonoterpene olefin limonene produced in Saccharomyces cerevisiae. Yeast, 2015. 32(1): p. 159-171.

26. Covington, B.C., J.A. McLean, and B.O. Bachmann, Comparative mass spectrometry-based metabolomics strategies for the investigation of microbial secondary metabolites. Natural Product Reports, 2017. 34(1): p. 6-24.

27. Causon, T.J. and S. Hann, Review of sample preparation strategies for MS-based metabolomic studies in industrial biotechnology. Analytica Chimica Acta, 2016. 938: p. 18-32.

-228. Li, Z.C., et al., Integrating MS1 and MS2 Scans in High-Resolution Parallel Reaction Monitoring Assays for Targeted Metabolite Quantification and Dynamic C-13-Labeling Metabolism Analysis. Analytical Chemistry, 2017. 89(1): p. 877-885.

Here, the authors present the application of parallel reaction monitoring in metabolomics, as a method to rapidly characterize the metabolic pathway reorganization observed in engineered $E$. coli strains.

29. Zhou, J.T., et al., Development and Evaluation of a Parallel Reaction Monitoring Strategy for Large-Scale Targeted Metabolomics Quantification. Analytical Chemistry, 2016. 88(8): p. 4478-4486.

30. Naz, S., et al., Development of a Liquid Chromatography High Resolution Mass Spectrometry Metabolomics Method with High Specificity for Metabolite Identification Using All Ion Fragmentation Acquisition. Analytical Chemistry, 2017. 89(15): p. 79337942.

••31. Ohtake, T., et al., Metabolomics-driven approach to solving a CoA imbalance for improved 1-butanol production in Escherichia coli. Metabolic Engineering, 2017. 41: p. 135-143. Using global metabolic profiling, the authors identify a non-obvious new target for enhancing the production of a desired end compound in engineered microbes.

-32. Noguchi, S., et al., Quantitative target analysis and kinetic profiling of acyl-CoAs reveal the rate-limiting step in cyanobacterial 1-butanol production. Metabolomics, 2016. 12(2). A combination of label-free global metabolite profiling and stable-isotope labelled fluxomics revealed new potential rate-limiting steps in a well-studied metabolic pathway.

33. Liu, L.N., X.G. Duan, and J. Wu, Modulating the direction of carbon flow in Escherichia coli to improve L-tryptophan production by inactivating the global regulator FruR. Journal of Biotechnology, 2016. 231: p. 141-148.

$\bullet \bullet 34$. Rodriguez, A., et al., Plasmid-encoded biosynthetic genes alleviate metabolic disadvantages while increasing glucose conversion to shikimate in an engineered Escherichia coli strain. Biotechnology and Bioengineering, 2017. 114(6): p. 1319-1330. A combination of netabolomics profiling and fluxomics experiments provided detailed insights into the profound metabolic consequences of the overproduction of a key precursor for an important family of natural products.

35. Lai, B., et al., Quantitative analysis of aromatics for synthetic biology using liquid chromatography. Biotechnology Journal, 2017. 12(1): p. 1600269.

36. Teoh, S.T., et al., A metabolomics-based strategy for identification of gene targets for phenotype improvement and its application to 1-butanol tolerance in Saccharomyces cerevisiae. Biotechnology for Biofuels, 2015. 8.

37. Jensen, E.D., et al., Transcriptional reprogramming in yeast using dCas 9 and combinatorial gRNA strategies. Microbial Cell Factories, 2017. 16. 
38. Liu, H., et al., High titer mevalonate fermentation and its feeding as a building block for isoprenoids (isoprene and sabinene) production in engineered Escherichia coli. Process Biochemistry, 2017. 62: p. 1-9.

39. Kirby, J., et al., Engineering a functional 1-deoxy-D-xylulose 5-phosphate (DXP) pathway in Saccharomyces cerevisiae. Metabolic Engineering, 2016. 38: p. 494-503.

40. Quinn, R.A., et al., Molecular Networking As a Drug Discovery, Drug Metabolism, and Precision Medicine Strategy. Trends in Pharmacological Sciences, 2017. 38(2): p. 143154.

41. Wang, M.X., et al., Sharing and community curation of mass spectrometry data with Global Natural Products Social Molecular Networking. Nature Biotechnology, 2016. 34(8): p. 828-837.

42. Olivon, F., et al., MZmine 2 Data-Preprocessing To Enhance Molecular Networking Reliability. Analytical Chemistry, 2017. 89(15): p. 7836-7840.

43. Olivon, F., et al., Optimized experimental workflow for tandem mass spectrometry molecular networking in metabolomics. Analytical and Bioanalytical Chemistry, 2017. 409(24): p. 5767-5778.

-44. Mohimani, H., et al., Dereplication of peptidic natural products through database search of mass spectra. Nature Chemical Biology, 2017. 13(1): p. 30-37.

This study illustrates the power of computational metabolomics, introducing a new algorithm for the dereplication of natural products libraries using molecular networking strategies.

$\bullet \bullet 45$ Nguyen, D.D., et al., Indexing the Pseudomonas specialized metabolome enabled the discovery of poaeamide B and the bananamides. Nature Microbiology, 2017. 2(1). Metabolite networking enabled the rapid parallel exploration of the secondary metabolome of more than two hundred ecologically diverse strains of microbes, facilitating dereplication, while at the same time identifying structural relationships between newly discovered compounds.

46. Crusemann, M., et al., Prioritizing Natural Product Diversity in a Collection of 146 Bacterial Strains Based on Growth and Extraction Protocols. Journal of Natural Products, 2017. 80(3): p. 588-597.

47. von Eckardstein, L., et al., Total Synthesis and Biological Assessment of Novel Albicidins Discovered by Mass Spectrometric Networking. Chemistry-a European Journal, 2017. 23(61): p. 15316-15321.

48. Hartmann, A.C., et al., Meta-mass shift chemical profiling of metabolomes from coral reefs. Proceedings of the National Academy of Sciences of the United States of America, 2017. 114(44): p. 11685-11690.

49. Hoffman, M., et al., Homospermidine Lipids: A Compound Class Specifically Formed during Fruiting Body Formation of Myxococcus xanthus DK1622. Acs Chemical Biology, 2018. 13(1): p. 273-280.

50. Okada, B.K., et al., Mapping the Trimethoprim-Induced Secondary Metabolome of Burkholderia thailandensis. Acs Chemical Biology, 2016. 11(8): p. 2124-2130.

51. Brunk, E., et al., Characterizing Strain Variation in Engineered E.coli Using a Multi-OmicsBased Workflow. Cell Systems, 2016. 2(5): p. 335-346. 
Table 1

Glossary of analytical technologies

\begin{tabular}{|c|c|c|}
\hline Technique/Approach & Full Name & Description \\
\hline Metabolomics & - & $\begin{array}{l}\text { The untargeted, non-biased } \\
\text { detection and identification of all } \\
\text { low-molecular weight compounds } \\
\text { (metabolites) present within a } \\
\text { biological sample or system. }\end{array}$ \\
\hline MS & Mass Spectrometry & $\begin{array}{l}\text { Analytical technique based on the } \\
\text { ionisation of analytes (e.g., by DESI, } \\
\text { MALDI, PTR or SIFT; see below), the } \\
\text { subsequent separation of ions } \\
\text { according to mass/charge ratio, and } \\
\text { their detection and quantification. }\end{array}$ \\
\hline MSI & $\begin{array}{l}\text { Mass Spectrometry } \\
\text { Imaging }\end{array}$ & $\begin{array}{l}\text { Mass spectrometry is conducted in a } \\
\text { spatial manner thus permitting the } \\
\text { visualisation of the two-dimensional } \\
\text { localisation of analytes within a } \\
\text { sample, for example across a } \\
\text { microbial colony growing on an } \\
\text { agar plate. }\end{array}$ \\
\hline DESI-MS & $\begin{array}{l}\text { Desorption Electrospray } \\
\text { Ionization Mass } \\
\text { Spectrometry }\end{array}$ & $\begin{array}{l}\text { Ambient ionization technique using } \\
\text { a nebulized electrospray. Highly } \\
\text { charged microdroplets collect } \\
\text { analytes from the surface of the } \\
\text { sample prior to secondary droplets } \\
\text { carrying the analyte to the MS. This } \\
\text { ionization technique is particularly } \\
\text { suitable for MSI. }\end{array}$ \\
\hline IM-MS & $\begin{array}{l}\text { Ion Mobility Mass } \\
\text { Spectrometry }\end{array}$ & $\begin{array}{l}\text { A variant of MS, with additional } \\
\text { separation of ions according to the } \\
\text { time it takes for them to travel } \\
\text { through a drift tube with a } \\
\text { homogeneous, continuous electric } \\
\text { field in the presence of a neutral gas. } \\
\text { This leads to separation of ions } \\
\text { according to size and shape } \\
\text { (collision cross section), } \\
\text { complementing the mass/charge } \\
\text { information available in traditional } \\
\text { MS }\end{array}$ \\
\hline MALDI-MS & $\begin{array}{l}\text { Matrix Assisted Laser } \\
\text { Desorption Ionization } \\
\text { Mass Spectrometry }\end{array}$ & $\begin{array}{l}\text { Ionization approach whereby a } \\
\text { matrix (an energy-absorbing small } \\
\text { organic compound) is applied } \\
\text { to/mixed with a sample. A laser } \\
\text { applied to the matrix:sample mix } \\
\text { excites the matrix molecules and } \\
\text { leads to the generation of volatilized } \\
\text { ions which subsequently enter the } \\
\text { MS. This technique is suitable for } \\
\text { MSI. }\end{array}$ \\
\hline PTR-MS & $\begin{array}{l}\text { Proton Transfer } \\
\text { Reaction Mass }\end{array}$ & $\begin{array}{l}\text { A soft ionization technique using an } \\
\text { ion beam of protonated water }\end{array}$ \\
\hline
\end{tabular}




\begin{tabular}{|c|c|c|}
\hline & Spectrometry & $\begin{array}{l}\text { molecules, } \mathrm{H}_{3} \mathrm{O}^{+} \text {, as an ion source to } \\
\text { protonate (and thus ionize) volatile } \\
\text { analytes. This technique permits for } \\
\text { real-time monitoring of organic } \\
\text { molecules in the gas phase. }\end{array}$ \\
\hline SIFT-MS & $\begin{array}{l}\text { Selected-Ion Flow-Tube } \\
\text { Mass Spectrometry }\end{array}$ & $\begin{array}{l}\text { Similar to PTR-MS, this soft } \\
\text { ionisation technique uses precursor } \\
\text { ions in the gas phase to ionize } \\
\text { volatile analytes. The precursor ions } \\
\text { are generated by a microwave } \\
\text { plasma ion source, and a single ion } \\
\text { species can be selected }\left(\mathrm{H}_{3} \mathrm{O}^{+}, \mathrm{NO}^{+}\right. \\
\text {or } \mathrm{O}_{2}^{-} \text {) to perform as reactant ion. } \\
\text { Neutral volatile analyte molecules } \\
\text { react with the precursor ions and } \\
\text { undergo ionization. This technique } \\
\text { permits for real-time monitoring in } \\
\text { the gas phase. }\end{array}$ \\
\hline Molecular Networking & - & $\begin{array}{l}\text { A computational method for MS } \\
\text { data analysis that allows for the } \\
\text { identification of sets of spectra from } \\
\text { chemically related molecules } \\
\text { ("spectral networks"), based on } \\
\text { similarities in molecular } \\
\text { fragmentation patterns, even in the } \\
\text { cases when the spectra are not } \\
\text { matched to any known compounds. }\end{array}$ \\
\hline
\end{tabular}

